\title{
Role of distributed Bragg reflection in photonic-crystal optical waveguides
}

\author{
Ali Adibi \\ Georgia Institute of Technology, School of Electrical and Computer Engineering, Atlanta, Georgia 30332 \\ Yong Xu, Reginald K. Lee, Marko Loncar, Amnon Yariv, and Axel Scherer \\ California Institute of Technology, Departments of Electrical Engineering and Applied Physics, Pasadena, California 91125
}

(Received 1 March 2001; published 6 July 2001)

\begin{abstract}
We show that the properties of the confined modes of a photonic band-gap (PBG) waveguide can be calculated with good accuracy by replacing it with an effective corrugated waveguide that represents only the structure in the vicinity of the middle slab. Such a replacement is helpful in the design of the PBG waveguides as well as in the understanding and analysis of the coupling of different waveguides.
\end{abstract}

DOI: 10.1103/PhysRevB.64.041102

PACS number(s): 42.70.Qs, 42.79.Gn

Photonic crystals have inspired a great deal of interest recently due to their potential for controlling the propagation of light. Photonic crystals with line defects can be used for guiding light. Photonic-crystal waveguides [or photonic band-gap (PBG) waveguides] are important for connecting photonic crystal optical devices. Guiding light through sharp bends has been recently proposed ${ }^{1}$ and demonstrated at microwave $^{2}$ and optical ${ }^{3}$ frequencies. The presence of the periodic structures in the guiding direction of PBG waveguides results in the distributed Bragg reflection (DBR) of the guided mode. It was recently proposed ${ }^{4,5}$ that the guiding of light in dielectric-core PBG waveguides is mainly controlled by total internal reflection and distributed Bragg reflection (DBR). In this paper, we investigate the effect of DBR on planar waveguides made by placing a thin dielectric slab between two PBG mirrors made of two-dimensional lattices of air columns in a dielectric material. Choosing the thickness of the middle slab appropriately results in singlemode propagation in the waveguide. ${ }^{4} \mathrm{We}$ show that several properties of the modes of a PBG waveguide depend on the DBR from the two rows of the photonic crystal that are ad- jacent to the middle slab. We also discuss the effect of DBR on the coupling of a dielectric slab waveguide to a PBG waveguide.

Figure 1(a) shows one unit cell of a PBG waveguide made by placing a dielectric slab of thickness $d$ between two PBG mirrors, each consisting of a two-dimensional square lattice of air columns in GaAs. The period of the square lattice and the radius of each air column are $a$ and $r=0.45 a$, respectively. Since the guided mode of the PBG waveguide is mainly confined in the middle slab, an important role is played by the two rows of the air columns that are adjacent to (one on each side of) the middle slab. Figure 1(b) shows an equivalent corrugated waveguide made by reducing the thickness of each PBG mirror in Fig. 1(a) to $r$. This results in a one-dimensional waveguide with period $a$. To analyze the guiding structures, we used a computer code based on the two-dimensional finite difference time domain (2D-FDTD) method. ${ }^{6}$ To compute the dispersion diagrams of the guided modes, we used an order- $N$ spectral method. ${ }^{7}$ In the calculations of this paper, the speed of light in vacuum $(c)$ is normalized to 1 , and all spatial dimensions are in the units of
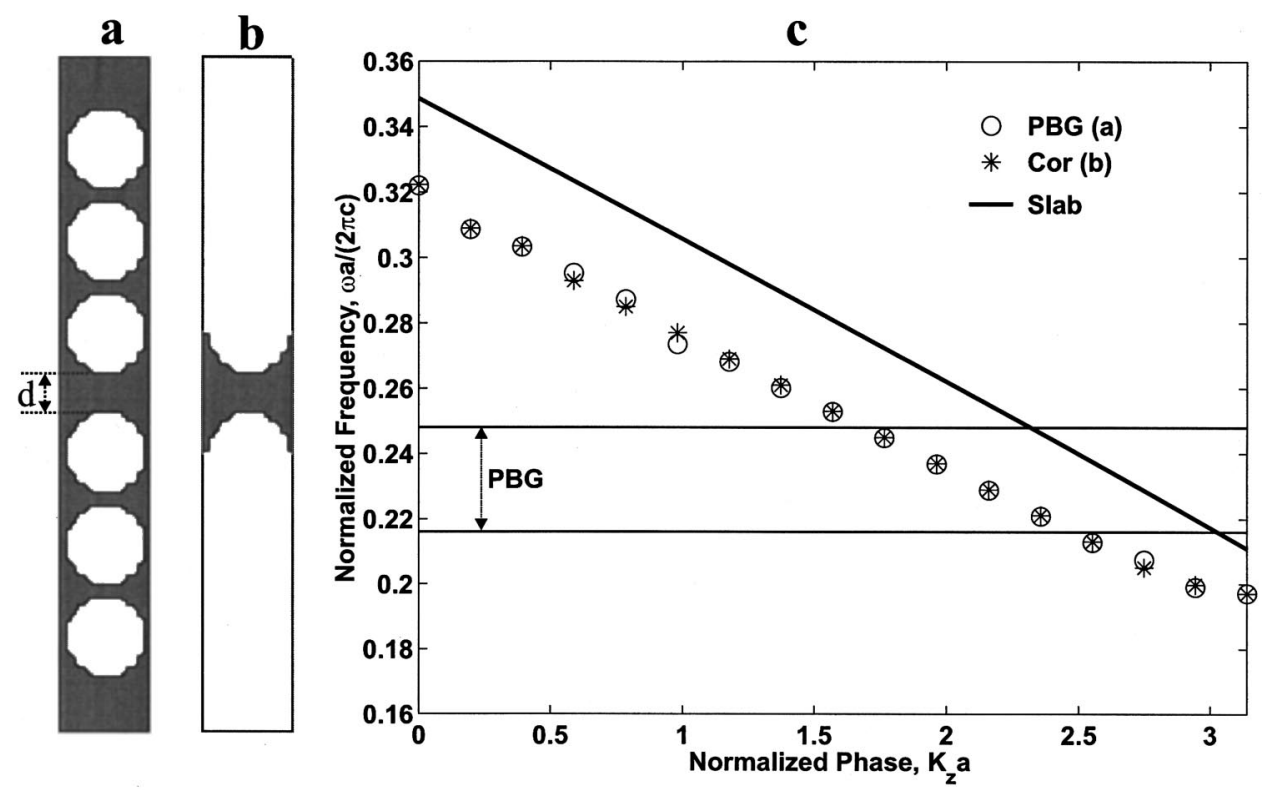

FIG. 1. (a) A square-lattice PBG waveguide, (b) the equivalent corrugated waveguide, and (c) dispersion diagrams of the fundamental modes of the two waveguides shown in parts (a) and (b) along with the dispersion diagram of a dielectric slab waveguide. All three waveguides have similar slab thicknesses $(d=9$ calculation cells) and relative permittivities $\left(\epsilon_{S}=12.96\right)$. The cladding region of the slab waveguide is air $(\epsilon=1)$. The period of the square lattice and the radius of each air column in the PBG waveguide in (a) are $a=24$ calculation cells, and $r=0.45 a$, respectively. 
calculation cells. To compute the band structure of the PBG waveguide and the dispersion diagrams of the guided modes, we used Bloch boundary conditions on the left and right sides and perfectly matched layers (PML's) (Ref. 8) on the top and bottom of the structures shown in Figs. 1(a) and (b).

Figure 1(c) shows the dispersion diagrams for the fundamental TE modes (electric field normal to the computation plane) of the two waveguides shown in Figs. 1(a) and (b), as well as that of a conventional dielectric slab waveguide with the same slab thickness $(d)$. The dispersion curve of the slab waveguide mode in Fig. 1(c) is restricted to the first Brillouin zone of the slab waveguide with imposed artificial spatial periodicity the same as that of the PBG waveguide. The photonic band gap (PBG) of the two-dimensional photonic crystal of Fig. 1(a) is also shown in Fig. 1(c). As Fig. 1(c) shows, the dispersion diagram of the fundamental mode of the PBG waveguide is approximately the same as that of the fundamental mode of the equivalent corrugated waveguide. This suggests that the properties of the fundamental guided mode of the PBG waveguide are mainly controlled by the two rows, one on each side, of the photonic crystal that are adjacent to the middle slab. Therefore we can replace a PBG waveguide by its equivalent corrugated waveguide to obtain the approximate behavior. Such a replacement is also useful in obtaining approximate analytic formulas for the properties of the fundamental guided mode of a PBG waveguide, since the analysis of corrugated waveguides is straightforward. ${ }^{9}$

To show the power of the above method for replacing a PBG waveguide with its equivalent corrugated waveguide, we consider the serial coupling of a dielectric slab waveguide to a PBG waveguide [Fig. 2(a)] and to the equivalent corrugated waveguide [Fig. 2(b)]. The thickness $(d)$ and relative permittivity $\epsilon_{S}$ of the middle slab is the same for all three waveguides. To calculate the transmission spectrum from the slab waveguide to the PBG waveguide (or the corrugated waveguide), we used a pulsed Huygens source ${ }^{10}$ to excite the fundamental TE mode in the slab waveguide. We used perfectly matched layers (PMLs) at all boundaries of the structure and calculated the spectrum of the power transmitted through the PBG waveguide (or the corrugated waveguide) by taking the Fourier transform of the fields and then integrating the Poynting vector over a surface of 60 calculation cells centered at the middle of the PBG (or corrugated) waveguide slab at $L=8 a$ (after the eighth air column in the guiding direction). The power transmission coefficient was then calculated as the ratio of the transmitted and incident powers. ${ }^{4}$

Figure 2(c) shows the transmission spectra (ratio of the transmitted to incident power at different frequencies) for the two cases of coupling. As Fig. 2(c) shows, the transmission spectrum from the slab waveguide to the $\mathrm{PBG}$ waveguide is close to that from the slab waveguide to the equivalent corrugated waveguide at lower frequencies $[\omega a /(2 \pi c)$ $<0.27]$. The strong minimum at $\omega a /(2 \pi c) \simeq 0.18$ as well as the ripples in the high transmission region $[0.206$ $<\omega a /(2 \pi c)<0.27]$ are due to the DBR caused by the periodic structure on top and bottom of the middle slab. ${ }^{4}$ The mode of the PBG waveguide at higher frequencies $[\omega a /(2 \pi c)>0.27]$ is not confined to the middle slab due to
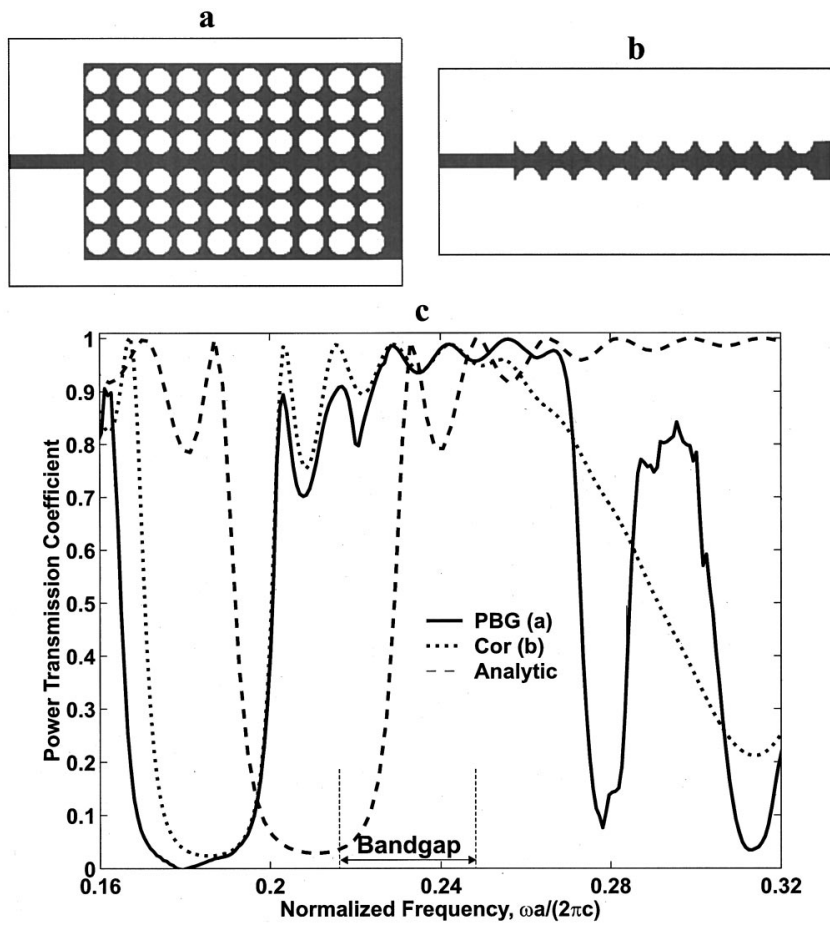

FIG. 2. Coupling of a slab waveguide to (a) a PBG waveguide, and (b) the equivalent corrugated waveguide. The properties of the three waveguides are summarized in the captions of Fig. 1. (c) Spectrum of power transmission coefficient (at $L=8 a$ ) from a dielectric slab waveguide to a PBG waveguide [part (a)], and to the equivalent corrugated waveguide [part (b)]. The approximate analytic formula for the latter [Eq. (7)] is also plotted. The photonic crystal in part (a) has a photonic band gap for $0.216<\omega a /(2 \pi c)$ $<0.248$ as shown in the figure.

the presence of other modes of the photonic crystal..$^{5}$ This results in a large difference between the transmission spectra for the two waveguides for $\omega a /(2 \pi c)>0.27$ as shown in Fig. 2(c). Therefore the replacement of a PBG waveguide with its equivalent corrugated waveguide is only valid for the modes that are well confined to the middle slab. This includes the lossless guided modes and excludes the modes of the photonic crystals that are extended in the structure.

We can find an approximate analytic formula for the power transmission coefficient shown in Fig. 2(c) by using the equivalent corrugated waveguide. To find such a formula, we use coupled-mode analysis, and follow the same steps as in Ref. 9. We approximate the corrugation on top of the middle slab (as shown in Fig. 3) by

$$
\begin{aligned}
\Delta \epsilon(x, z) & =\Delta n^{2}(x, z) \\
& =\left(\epsilon_{S}-1\right) \operatorname{rect}\left(\frac{x-d / 2-r / 2}{r}\right) \operatorname{rect}\left(\frac{z}{2 \tau(x)}\right),
\end{aligned}
$$

where $\operatorname{rect}(u)$ is the rectangle function

$$
\operatorname{rect}(u)=\left\{\begin{array}{cc}
1 & \text { for }|u|<\frac{1}{2} \\
0 & \text { otherwise }
\end{array}\right.
$$

and $\tau(x)$ is defined by 


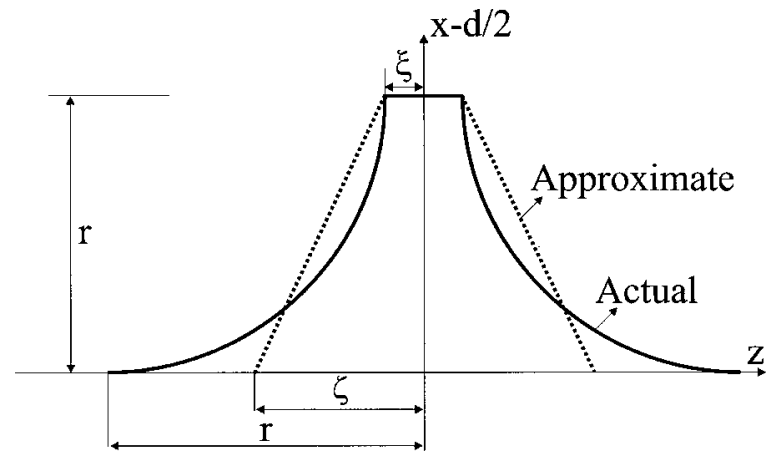

FIG. 3. Approximation of one period of the corrugation in Fig. 1(b). The circular arcs are replaced by straight lines. The corrugation is symmetric with respect to the vertical $(x-d / 2)$ axis. Note that $\xi=a / 2-r$, where $a$ is the period of the structure.

$$
\tau(x)=\xi+\zeta-\frac{\zeta}{r}(x-d / 2),
$$

with $\xi$ and $\zeta$ defined in Fig. 3. Here, we replaced each circular arc of the corrugation by a straight line. Therefore $\zeta$ can be varied to optimize the approximation. We can write a similar formula to model the corrugation at the bottom of the middle slab in Fig. 2(b). However, due to the symmetry of the structure, we only need to compute the effect of one of the corrugations and multiply the results by 2 .

Since the corrugation on the top of the structure is periodic (with period $a$ ) with respect to $z$, we can represent it with a Fourier series. The $l$ th (non-dc) term of the Fourier series of $\Delta \epsilon(x, z)$ with respect to $z$ is

$$
a_{1}(x)=\left(\epsilon_{S}-1\right) \operatorname{rect}\left(\frac{x-d / 2-r / 2}{r}\right) \frac{\sin [2 \pi l \tau(x) / a]}{\pi l} .
$$

The normalized electric field of the fundamental TE mode $\left(E_{y}\right)$ of the dielectric slab waveguide with slab thickness $d$ and slab relative permittivity $\epsilon_{S}$ is given by ${ }^{9}$

$$
E_{y}(x, z)=\left\{\begin{array}{cc}
A \exp [-p(x-d / 2)-i \beta z] & \text { for } x>d / 2 \\
A \frac{\cos (h x)}{\cos (h d / 2)} \exp [-i \beta z] & \text { for }|x|<d / 2, \\
A \exp [p(x+d / 2)-i \beta z] & \text { for } x,-d / 2
\end{array}\right.
$$

where $\beta^{2}=k_{0}^{2}+p^{2}=\epsilon_{S} k_{0}^{2}-h^{2}$ and $p d / 2=h d / 2 \tan (h d / 2)$. In these equations, $k_{0}=2 \pi / \lambda$ and $\beta$ are the propagation constant of a plane wave in free space and propagation constant of the fundamental mode of the slab waveguide in the $z$ direction, respectively. The amplitude of the normalized electric field $(A)$ is chosen to be

$$
A=\left[\frac{2 \omega \mu}{\beta} \frac{h^{2} p}{\left(h^{2}+p^{2}\right)(1+p d / 2)}\right]^{1 / 2} .
$$

This results in the following normalization condition of the mode

$$
\int_{-\infty}^{+\infty}\left|E_{y}(x, z)\right|^{2} d x=\frac{2 \omega \mu}{\beta} .
$$

Using Eqs. (4) and (5), we can calculate the coupling coefficient $\kappa$,

$$
\begin{aligned}
\kappa= & \frac{i \omega \epsilon_{0}}{4} \int_{-\infty}^{+\infty} a_{1}(x)\left|E_{y}(x, z)\right|^{2} d x \\
= & \frac{i \omega^{2} \mu \epsilon_{0}}{2 \beta} \frac{\epsilon_{S}-1}{\pi l} \frac{h^{2}}{\left(h^{2}+p^{2}\right)(1+p d / 2)} \\
& \times \frac{\sin \left[\frac{2 \pi l(\xi+\zeta)}{a}\right]+\left(\frac{\pi l \zeta}{\operatorname{arp}}\right)\left[\exp (-2 p r)-\cos \left(\frac{2 \pi l \zeta}{a}\right)\right]}{1+\left(\frac{\pi l \zeta}{\operatorname{arp}}\right)^{2}} .
\end{aligned}
$$

The power transmission coefficient $(T)$ calculated at $z$ $=L$ is

$$
T=\left|\frac{S}{-\Delta \beta \sinh (S L)+i S \cosh (S L)}\right|^{2},
$$

where $\Delta \beta=\beta-l \pi / a$ and $S=\sqrt{|\kappa|^{2}-\Delta \beta^{2}}$. The transmission coefficient $T$ calculated from Eq. (9) is also plotted in Fig. 2(c) as a function of normalized frequency. As Fig. 2(c) shows, the approximate analytic formula predicts the position of the DBR minimum with some error. However, it predicts the strength of the DBR minimum more accurately. The reason for the difference between the analytic formula and the numerical calculation of the power transmission coefficient at different frequencies is that the perturbation of the relative permittivity $\left(\epsilon_{S}-1=11.96\right)$ is not very small. Therefore the propagation constant of the fundamental mode $(\beta)$ in the corrugated waveguide is different from that of the dielectric slab waveguide. This can be seen from Fig. 1(c) where the dispersion diagram of the fundamental mode of the slab waveguide lies above that of the fundamental mode of the equivalent corrugated waveguide. We can obtain a better approximation by first calculating $\beta$ of the fundamental mode of the corrugated waveguide using the dispersion diagram of Fig. 1(c). Optimization of the corrugation approximation (by varying $\zeta$ in Fig. 3) can result in a better approximation, too.

The accuracy in the replacement of a PBG waveguide with the equivalent corrugated slab waveguide depends on the degree of mode confinement in the middle slab. This depends on the photonic crystal and the radius of the air columns. To show the applicability of the method to other PBG waveguides, we calculated the dispersion diagram of the fundamental mode of a PBG waveguide made by removing one row of air columns from a triangular lattice of air columns (lattice period $a$, radius of air column $\tau=0.3 a$ ). To obtain single-mode propagation within the photonic band gap, we increased the radii of the air columns that are adjacent to the middle slab to $r^{\prime}=0.4 a$ (Ref. 11) as shown in Fig. 4(a). The equivalent corrugated waveguide includes the 

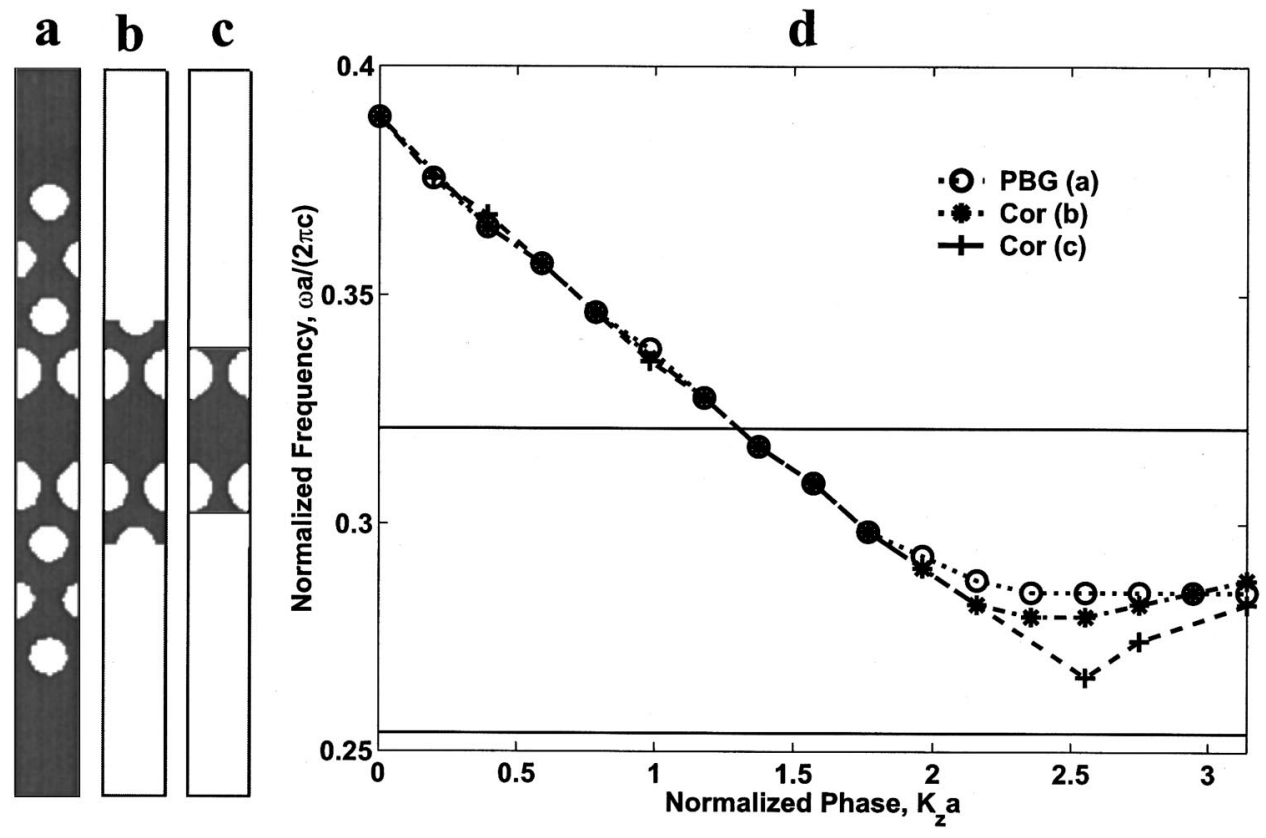

FIG. 4. (a) A triangular-lattice PBG waveguide, (b) an equivalent corrugated waveguide, (c) a more approximate corrugated waveguide, and (d) dispersion diagrams of the fundamental modes of the three waveguides shown in parts (a), (b), and (c). The PBG waveguide is made by removing one row of air columns from a triangular lattice of air columns in a dielectric material $\left(\epsilon_{S}=7.29\right)$. The radii of the air columns in the two rows adjacent to the middle slab are $r^{\prime}=0.4$. The radii of all other air columns are $r=0.3 a$. In these calculations, we used $a$ $=24$ calculation cells.

two layers of air columns that are adjacent to the middle slab (one on each side) as well as half of the second row of air columns on each side of the middle slab to conserve the properties of the triangular lattice in the corrugated waveguide [Fig. 4(b)]. The dispersion diagrams of the fundamental modes of the two waveguides are shown in Fig. 4(d). The similarity between the dispersion diagrams of the fundamental modes of the two waveguides is evident from Fig. 4(d). In Fig. 4(d), we have also shown the dispersion diagram for the fundamental mode of another corrugated waveguide made by keeping only the two rows of air column that are adjacent to the middle slab [Fig. 4(c)]. Such a corrugated waveguide does not conserve the triangular lattice of the air columns compared to the corrugated waveguide of Fig. 4(b). This explains the larger deviation of the dispersion diagram of the latter corrugated waveguide [Fig. 4(c)] from the actual dispersion diagram of the $\mathrm{PBG}$ waveguide compared to the former corrugated waveguide [Fig. 4(b)]. Calculation of the dispersion diagram for a similar PBG waveguide with $r$ $=r^{\prime}=0.3 a$ shows similar results.

In conclusion, we showed that the properties of the confined modes of a PBG waveguide can be calculated with good accuracy by replacing it with an effective corrugated waveguide that represents only the structure in the vicinity of the middle slab. Replacing a PBG waveguide with its equivalent corrugated waveguide is an effective tool for understanding and designing PBG waveguides. It reduces the computation time of the structures considerably. It enables us to derive approximate analytic formulas for the important properties of the PBG waveguides. Such formulas are usually the best starting points in optimizing the structures. Fine tuning of the optimization can then be performed by full numerical simulation of the actual PBG waveguide.

This work was supported in part by the Air Force Office of Scientific Research under contract AFOSR-61557, and by the Office of Naval Research (Y. S. Park).
${ }^{1}$ A. Mekis, J. C. Chen, I. Kurland, S. Fan, P. R. Villeneuve, and J. D. Joannopoulos, Phys. Rev. Lett. 77, 3787 (1996).

${ }^{2}$ S. Lin, E. Chow, V. Hietala, P. R. Villeneuve, and J. D. Joannopoulos, Science 282, 274 (1998).

${ }^{3}$ T. Baba, N. Fukaya, and J. Yonekura, Electron. Lett. 35, 654 (1999).

${ }^{4}$ A. Adibi, Y. Xu, R. K. Lee, A. Yariv, and A. Scherer, J. Lightwave Technol. 18, 1554 (2000).

${ }^{5}$ A. Adibi, Y. Xu, R. K. Lee, A. Yariv, and A. Scherer, Phys. Rev. B 64, 33308 (2001).
${ }^{6}$ K. S. Yee, IEEE Trans. Antennas Propag. AP-14, 302 (1966).

${ }^{7}$ C. T. Chan, Q. L. Yu, and K. M. Ho, Phys. Rev. B 51, 16635 (1995).

${ }^{8}$ J. P. Berenger, J. Comput. Phys. 114, 185 (1994).

${ }^{9}$ A. Yariv, Optical Electronics in Modern Communications (Oxford University Press, New York, 1996).

${ }^{10}$ D. E. Merewether, R. Fisher, and F. W. Smith, IEEE Trans. Nucl. Sci. NS-27, 1829 (1980).

${ }^{11}$ A. Adibi, R. K. Lee, Y. Xu, A. Yariv, and A. Scherer, Electron. Lett. 36, 1376-1378 (2000). 